\title{
Fluoxetine-Induced Sleep Disturbance in Depressed Patients
}

\author{
Cynthia M. Dorsey, Ph.D., Scott E. Lukas, Ph.D., and Steven L. Cunningham, R.PSG.T.
}

\begin{abstract}
Abnormal polysomnographic (PSG) features, most notably increased electromyographic (EMG) tone and eye movements during non-REM sleep have been observed during sleep in fluoxetine-treated depressed patients. However, the relationship between these PSG features and sleep disruption is unclear. Nine depressed patients trented with 10 to $80 \mathrm{mg}$ of fluoxetine and six unmedicated, depressed patients were studied polysomnographically on two consecutive nights during which sleep parameters, transient arousals, and eye movements were measured. The fluoxetine group experienced a lower-average sleep
\end{abstract}

KEY WORDS: Fluoxetine; Arousals; PLMD; Nocturnal Myoclonus; Insomnia

Fluoxetine is a selective serotonin reuptake inhibitor that is prescribed frequently to treat psychiatric conditions such as depression and obsessive compulsive disorder. Patients treated with fluoxetine often complain of insomnia $(7 \%-22 \%)$ or daytime drowsiness $(10 \%-15 \%)$ (Stark and Hardison 1985; Wernicke 1985; Cooper 1988), and insomnia is associated more often with fluoxetine than with other tricyclic antidepressant medications such as imipramine, amitriptyline, and doxepin (Wernicke 1985; Cooper 1988). The mechanism(s) by which fluoxetine disturbs sleep is unclear, although polysomnographic features associated with fluoxetine have been described (Keck et al. 1991; Dorsey et al. 1992a; Schenck et al. 1992; Armitage et al. 1994).

These reports indicate that depressed patients expe-

From the Sleep Disorders Center (CMD, SEL, SLC) and the Alcohol and Drug Abuse Research Center (SEL), McLean Hospital, Harvard Medical School, Belmont, MA.

Address correspondence to: Dr. Cynthia M. Dorsey, Sleep Disorders Center and Sleep Research Program, McLean Hospital, 115 Mill Street, Belmont, MA 02178 .

Received May 1, 1995; revised July 22, 1995; accepted July 28, 1995. efficiency index (SEI) and significantly more eye movements and arousals during non-REM sleep than the control group. Eye movement and arousal counts were significantly correlated. In addition, clinically significant periodic limb movement disorder (PLMD) was observed in $44 \%$ of the fluoxetine-treated group versus none of the control group. We conclude that a higher incidence of PLMD and frequent transient arousals associated with eye movements may be responsible in part for the complaint of insomnia made by patients treated with fluoxetine.

[Neuropsychopharmacology 14:437-442, 1996]

rience frequent eye movements and elevated muscle tone during sleep. Our group initially described a pattern of medium-to-fast eye movements during nonREM sleep and elevated muscle tone in seven depressed patients treated with fluoxetine (Keck et al. 1991) and later in depressed patients on fluoxetine compared with those treated with other antidepressant medications (Dorsey et al. 1992a). Schenck et al. (1992) reported similar findings in a large retrospective study and described a case of REM behavior disorder associated with fluoxetine. Armitage et al. (1994) quantified increases in eye movements and muscle tone during sleep after treatment with fluoxetine by measuring eye movement counts and changes in electrooculographic (EOG) and electromyographic (EMG) amplitude. However, none of these studies specifically explored measures of EEG sleep continuity in relation to treatment with fluoxetine. The findings reported in these previous studies are descriptive features of polysomnographic (PSG) effects of fluoxetine on sleep but do not explain the subjective complaint of insomnia.

Antidepressant medications also have been found to cause or exacerbate periodic leg movements during sleep, disrupting sleep continuity, and causing insom- 
nia (Guilleminault et al. 1975; Ware 1983; Myers et al. 1986). However, diagnosis and interpretation of myoclonus are problematic in that myoclonus has been defined in a number of different ways by different investigators, using various criteria regarding time of day, frequency and duration of events, localization, and severity. Very few studies in which an association between myoclonus and antidepressants was made involved use of PSG monitoring to provide objective measures of nocturnal myoclonus or periodic limb movements during sleep. Myoclonus has been defined by subjective complaint and/or observer report as irregular muscular contractions, sudden facial twitches or jerks in upper or lower extremities that may occur exclusively during the daytime or during sleep as well (Lippmann et al. 1977; Casas et al. 1987; Garvey and Tollefson 1987). Casas et al. (1987) noted that involvement of the upper limbs or trunk or facial dyskinesias is less frequent than that of leg movements in myoclonus seen in patients treated with clomipramine.

In studies in which PSG monitoring was employed to document nocturnal myoclonus, periodicity and frequency criteria are either not specified or very different from one another (Guilleminault et al. 1975; Ware 1983; Myers et al. 1986; Askenasy and Yahr 1988; Forstl and Pohlmann-Eden 1990). Guilleminault et al. (1975) described nocturnal myoclonus, jerking during sleep, particularly at the onset of sleep in stages one and two sleep, chiefly involving the lower limbs. Askenasy and Jahr (1988) described a "switch myoclonus" that had very different features, characterized by movements of the lower limbs that occurred only at sleep onset and at the end of sleep at a rate of once per second. Ware (1983) referred to "myoclonic events" and Forstl and Pohlman-Eden (1990) to "bilateral myoclonic limb jerks," but neither described criteria used to define these terms.

The present study was conducted to characterize the specific disturbances of EEG sleep continuity that may contribute directly to the complaint of insomnia and sleepiness made by depressed patients treated with fluoxetine. The descriptive features identified in prior studies are readdressed here in terms of their relationship to EEG arousals and awakenings from sleep. The incidence of periodic limb movement disorder (PLMD), a sleep disorder that can cause EEG sleep disruption and a subsequent complaint of insomnia also was measured because a greater-than-normal incidence of PLMD in young adults treated with fluoxetine has been noted (Dorsey et al. 1992; Armitage et al. 1994).

\section{METHODS}

\section{Subjects}

Nine inpatients (two male, seven female) with a diagnosis of primary depression (DSM-III-R), who had been treated only with fluoxetine for at least 2 weeks and up to 1 year, were referred to the Sleep Disorders Center for evaluation of complaint of sleep-maintenance insomnia $(n=3)$, morning fatigue $(n=3)$, or both of these complaints $(n=3)$. The sleep symptoms began in the context of treatment with fluoxetine (within 1 to 3 weeks after treatment began or after an increase in dose). Four of the nine patients had an additional diagnosis of eating disorder (bulimia nervosa). Six nonmedicated inpatients (two male, four female) who were diagnosed with depression using the Structured Clinical Interview for DSM-III-R (SCID) (Spitzer et al. 1987) and Hamilton Depression Rating Scale (Hamilton 1960) and also had complaints of sleep maintenance insomnia and/or daytime fatigue, although not as severe as those of the treatment group, constituted the control group. The severity of depressive symptoms within the two groups was considered to be comparable based on the clinician's (CMD) review of interview and rating scale results. The mean ages of the fluoxetine and depressed control groups were $25.3 \pm 6.5$ and $23.4 \pm 5.9$, respectively. Individuals with a history of neurological or other medical or psychiatric illness, or primary sleep disorder defined by International Classification of Sleep Disorders (ICSD) criteria (Diagnostic Classification Steering Committee 1990) were excluded.

\section{Procedure}

Each fluoxetine and control patient underwent two consecutive nights of PSG monitoring. Data from the second night are used for the analysis in this study. The first night served as a screening night to rule out primary physiological sleep disorders. Standard electrode placement and scoring criteria (Rechtschaffen and Kales 1968) were used, including bilateral EMG of the anterior tibialis to monitor leg movements. Sleep scoring on an epoch-by-epoch basis was performed blind by two independent raters with $90 \%$ agreement based on standard criteria (Rechtschaffen and Kales 1968).

Nocturnal arousal count was determined for all patient polysomnograms based on blind scoring. Arousals were defined as "abrupt shifts in EEG frequency which may include theta, alpha and/or frequencies greater than $16 \mathrm{~Hz}$ but not spindles" subject to the rules specified within the preliminary report of the Atlas Task Force of the American Sleep Disorders Association (Atlas Task Force 1992). Arousal indices (number arousals per hour of sleep) were computed for total sleep time and for individual sleep stages (number of arousals per hour per sleep stage). Eye movements greater than $50 \mu \mathrm{V}$ in amplitude (baseline to peak) were scored throughout sleep and wake. An eye movement index (EMI) was computed for total sleep time and for sleep during each sleep stage as described for arousals.

All 15 polysomnograms also were subjected to blind 
ratings by two independent evaluators with $91 \%$ agreement for the presence or absence of clinically significant periodic leg movements ( $>$ five events per hour of sleep). The presence of PLMs were determined using duration, periodicity, and interval criteria established by Coleman (1982) and incorporated in the definition of PLMD (Diagnostic Classification Steering Committee 1990). These included: 0.5 - to 5 -seconds' duration, four or more consecutive periodic movements required for minimal analysis, and movements separated by an interval $>5$ and $<90$ seconds. The average of PLMD indices from the two raters are reported. Only periodic limb movement (PLM) events associated with, and clearly occurring prior to, nocturnal arousal were scored. Nocturnal arousals were as previously defined.

\section{Data Analysis}

Arousal indices (AIs) and eye movement indices (EMIs) during total sleep, non-REM sleep, and each sleep stage were the primary dependent variables of interest. Unpaired, one-tailed $t$-tests were performed to compare sleep continuity measures and arousal indices between fluoxetine and control group. T-tests also were performed to compare EMIs between groups for each sleep stage, total sleep time, and wake time. Unpaired twotailed tests were used to compare sleep stage parameters between groups because no a priori expectations about group differences in sleep stages were hypothesized. Specific correlations were performed to assess the relationships between arousals and eye movements for total sleep time, non-REM sleep, and individual sleep stage. Fisher's $r$ to $z$ significance levels of $p<.001, .01$, and .05 are reported. $\mathrm{Chi}^{2}$ analysis was performed to test the difference between groups in frequency of occurrence of PLMs.

\section{RESULTS}

Demographics and sleep stage parameters for both depressed control and treatment groups are shown in Table 1. The REM onset latency was significantly longer for the fluoxetine group versus the depressed control group $(t=4.4, p<.001)$. The sleep efficiency index (SEI) was significantly lower for the fluoxetine group $(t=1.8$, $p<.05)$. There were no other differences between groups in the time spent in bed, total sleep time, sleep latency, or any sleep stage parameter. Arousal indices were computed as described in the Procedures section

Table 1. Demographic and Objective Sleep Data for Fluoxetine-Treated and Control Patients

\begin{tabular}{|c|c|c|c|c|c|c|c|c|c|c|c|c|}
\hline \multirow[b]{2}{*}{ Patient } & \multirow[b]{2}{*}{ Age } & \multirow[b]{2}{*}{ Dose } & \multirow[b]{2}{*}{$\begin{array}{l}\text { PLM } \\
\text { Index }\end{array}$} & \multirow[b]{2}{*}{ SEI } & \multirow[b]{2}{*}{$\begin{array}{c}\text { TST } \\
\text { (min.) }\end{array}$} & \multirow[b]{2}{*}{$\begin{array}{c}\text { SOL } \\
\text { (min.) }\end{array}$} & \multirow[b]{2}{*}{$\begin{array}{l}\text { WASO } \\
\text { (min.) }\end{array}$} & \multicolumn{5}{|c|}{ Sleep Stages } \\
\hline & & & & & & & & $\begin{array}{c}1 \\
(\%)\end{array}$ & $\begin{array}{c}2 \\
(\%)\end{array}$ & $\begin{array}{c}\text { SWS } \\
(\%)\end{array}$ & $\begin{array}{c}\text { REM } \\
(\%)\end{array}$ & $\begin{array}{l}\text { ROL } \\
\text { (min.) }\end{array}$ \\
\hline \multicolumn{13}{|l|}{ Fluoxetine } \\
\hline F01 & 27 & 10 & 7.9 & 81.8 & 352.0 & 47.0 & 31.5 & 12.2 & 54.8 & 12.4 & 20.6 & 146.0 \\
\hline $\mathrm{F} 02$ & 22 & 20 & - & 88.4 & 380.5 & 25.5 & 22.0 & 9.4 & 60.7 & 15.2 & 14.7 & 187.5 \\
\hline F03 & 20 & 40 & - & 91.4 & 422.5 & 11.0 & 29.0 & 9.6 & 46.6 & 22.7 & 21.1 & 282.0 \\
\hline F04 & 21 & 40 & - & 91.1 & 331.5 & 0.5 & 32.0 & 4.7 & 39.5 & 27.0 & 28.8 & 99.5 \\
\hline F05 & 19 & 60 & - & 91.3 & 399.5 & 0.5 & 37.5 & 14.0 & 58.8 & 3.9 & 23.3 & 146.5 \\
\hline F06 & 25 & 80 & 9.0 & 84.4 & 380.5 & 6.0 & 64.5 & 11.2 & 48.1 & 20.4 & 20.4 & 124.5 \\
\hline $\mathrm{F} 07$ & 17 & 80 & - & 93.1 & 371.0 & 23.5 & 4.0 & 3.2 & 56.2 & 15.5 & 25.1 & 139.0 \\
\hline F08 & 37 & 20 & 15.0 & 84.8 & 334.6 & 15.0 & 45.0 & 4.5 & 54.5 & 14.1 & 26.9 & 128.0 \\
\hline F09 & 23 & 40 & 8.0 & 80.3 & 301.5 & 46.0 & 28.0 & 15.4 & 57.7 & 7.3 & 19.6 & 176.0 \\
\hline Mean & 23.4 & & 10.0 & $87.4^{a}$ & 363.7 & 19.4 & 32.6 & 9.4 & 53.0 & 15.4 & 22.3 & $158.8^{b}$ \\
\hline $\mathrm{SD}$ & 5.9 & & & 4.7 & 36.9 & 17.7 & 16.4 & 4.4 & 6.9 & 7.3 & 4.3 & 53.2 \\
\hline \multicolumn{13}{|l|}{ Control } \\
\hline C01 & 37 & - & - & 97.3 & 382.0 & 7.0 & 3.5 & 19.1 & 49.5 & 5.8 & 25.7 & 67.5 \\
\hline $\mathrm{CO} 2$ & 21 & - & - & 94.1 & 411.0 & 21.5 & 4.5 & 3.2 & 60.6 & 17.2 & 19.1 & 50.5 \\
\hline $\mathrm{CO3}$ & 29 & - & - & 84.7 & 374.0 & 12.0 & 55.5 & 5.9 & 57.6 & 15.8 & 20.7 & 72.5 \\
\hline $\mathrm{C} 04$ & 23 & - & - & 89.4 & 396.5 & 5.0 & 42.0 & 2.1 & 47.2 & 32.5 & 18.2 & 51.5 \\
\hline $\mathrm{CO5}$ & 21 & - & - & 93.3 & 394.0 & 9.0 & 19.5 & 6.9 & 48.0 & 20.4 & 24.7 & 73.0 \\
\hline $\mathrm{C} 06$ & 21 & - & - & 91.8 & 331.6 & 6.7 & 23.0 & 4.7 & 46.7 & 27.1 & 21.4 & 55.3 \\
\hline Mean & 25.3 & & & 91.8 & 381.5 & 10.2 & 24.7 & 7.0 & 51.6 & 19.8 & 21.6 & 61.7 \\
\hline $\mathrm{SD}$ & 6.5 & & & 4.3 & 27.6 & 6.0 & 20.7 & 6.2 & 5.9 & 9.3 & 3.0 & 10.5 \\
\hline
\end{tabular}

PLM: Periodic leg movement; SEI: sleep efficiency index; TST: total sleep time; SOL: sleep onset latency; WASO: wake after sleep onset; SWS: slow wave sleep; REM: rapid eye movement sleep; ROL: REM onset latency.

"Significantly different from control group, $p<.05$.

${ }^{b} p<.001$ 
Table 2. Arousal Data for Fluoxetine $(n=9)$ and Control $(n=6)$ Patients

\begin{tabular}{lcccccc}
\hline & \multicolumn{7}{c}{ Mean Arousal Index (AI) } & & \\
Sleep Stage & Control & (SD) & Fluoxetine & (SD) & $t$ & $p$ \\
\hline Stage 1 & 35.9 & $(21.3)$ & 26.6 & $(14.3)$ & -1.0 & NS \\
Stage 2 & 10.6 & $(4.8)$ & 20.2 & $(4.7)$ & 3.8 & $<.01$ \\
SWS & 4.3 & $(3.4)$ & 9.7 & $(5.5)$ & 2.1 & $<.05$ \\
REM & 17.9 & $(7.3)$ & 22.4 & $(7.9)$ & 1.1 & NS \\
NREM & 10.4 & $(3.9)$ & 18.4 & $(3.7)$ & 4.0 & $<.001$ \\
TST & 11.9 & $(4.2)$ & 19.0 & $(3.5)$ & 3.7 & $<.01$ \\
\hline
\end{tabular}

SWS: Slow wave sleep; REM: rapid eye movement sleep; NREM: non-REM sleep; TST: total sleep time.

for total sleep time and sleep stage. Results from $t$-tests indicated significantly greater mean arousal indices in the fluoxetine group during total sleep time and nonREM sleep. Analysis of arousal indices from individual sleep stages showed group differences for stages 2, slow-wave sleep, non-REM sleep, and total sleep time, but not for REM and stage-1 sleep (Table 2).

$T$-tests to compare eye movements between groups showed that both eye movement count $>50 \mu \mathrm{V}$ and mean EMI were significantly greater during wake time in bed, total sleep time, and non-REM sleep for the fluoxetine treatment group versus the nonmedicated depressed group (Table 3). Interestingly, there was no group difference between eye movement count or index for REM sleep. As is illustrated in Figure 1, there was no difference in the EMI between controls and patients taking less than $40 \mathrm{mg}$ of fluoxetine.

Eye movement count and arousal count were significantly correlated for total sleep time $(r=.58, p<.05)$, non-REM sleep $(r=.67, p<.01)$, and stage-2 sleep $(r=$ $.76, p<.001)$. Correlations in stages 1 , SWS, and REM sleep were not statistically significant based on Fisher's $r$ to $z p$ values.

Four of the nine patients treated with fluoxetine $(44 \%)$ were rated as having clinically significant PLMD; none of the six control subjects had PLMD. The PLM indices for the fluoxetine patients who showed PLMD were low $(<15$ events per hour of sleep, mean PLM index $=10$ events per hour), indicating mild PLMD. $\mathrm{Chi}^{2}$ test showed a trend for a significant difference between groups in frequency of occurrence of clinically significant PLMD $\left(x^{2}=3.64, p=.056\right)$. The four patients who had PLMD were treated with 10, 20,40, and $80 \mathrm{mg}$ of fluoxetine, respectively. Some aperiodic leg movements also were observed in the sleep recordings of fluoxetine patients, but these movements did not meet criteria for scoring PLMD. Elevation of overall muscle tone was apparent in all fluoxetine recordings. Arousals that clearly occurred secondary to PLMD were not included in the spontaneous arousal indices.

\section{DISCUSSION}

Overall, fluoxetine was associated with a greater number of arousals from sleep, specifically from non-REM sleep and most notably from stage-2 sleep. This subtle measure of sleep disruption seemed to reflect a treatment effect, as did a conventional sleep scoring measure of continuity (SEI). However, measures of sleep continuity were not made on fluoxetine-treated patients prior to treatment. Therefore, we are unable to determine with certainty the extent to which these features can be attributable to fluoxetine. Also, the fact that four of the nine depressed fluoxetine-treated patients had an additional diagnosis of bulimia nervosa may represent

Table 3. Eye Movement Data for Fluoxetine $(n=9)$ and Control $(n=6)$ Patients

\begin{tabular}{lrrrrrl}
\hline & \multicolumn{7}{c}{ Mean Eye Movement Index (EMI) } & & \\
\cline { 2 - 5 } Sleep Stage & Control & (SD) & Fluoxetine & (SD) & $\boldsymbol{t}$ & $\boldsymbol{p}$ \\
\hline Wake & 198.3 & $(109.1)$ & 380.0 & $(160.9)$ & 2.4 & $<.05$ \\
Stage 1 & 142.9 & $(77.0)$ & 281.1 & $(199.3)$ & 1.6 & NS \\
Stage 2 & 12.4 & $(8.7)$ & 99.7 & $(73.1)$ & 2.9 & $<.01$ \\
SWS & 1.0 & $(1.8)$ & 75.6 & $(75.4)$ & 2.4 & $<.05$ \\
REM & 238.3 & $(94.7)$ & 315.3 & $(115.4)$ & 1.4 & NS \\
NREM & 16.5 & $(11.1)$ & 109.4 & $(67.6)$ & 3.3 & $<.01$ \\
TST & 65.5 & $(33.3)$ & 156.2 & $(81.5)$ & 2.6 & $<.05$ \\
\hline
\end{tabular}

SWS: Slow wave sleep; REM: rapid eye movement sleep; VREM: non-REM sleep; TST: total sleep time 


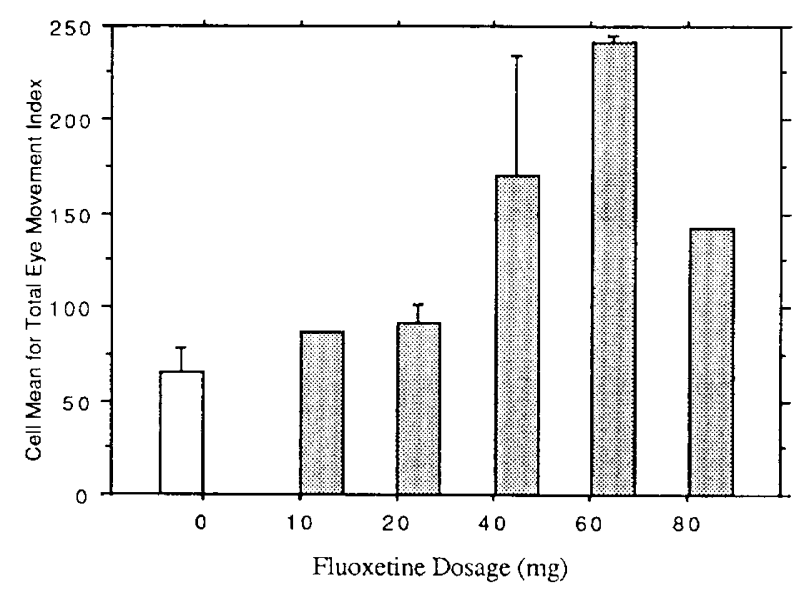

Figure 1. Eye movement index (EMI) during all sleep stages as a function of fluoxetine dose. Data represent mean \pm s.d. of 6 control and 9 fluoxetine patients. ( $\square$ control; $\square$ fluoxetine).

a clinical difference between groups, although continuity and other sleep measures for this subset of patients with bulimia did not differ from those of the rest of the group treated with fluoxetine.

Eye movements occurred tonically only in the treatment group and far more frequently in every stage of sleep. Both arousals and eye movements were significantly increased during stage- 2 and SWS but not during stages-1 and REM sleep for the fluoxetine versus the unmedicated group. These results are in contrast to a previous report of "dramatic" eye movement increases in REM sleep and no increase in stage-2 sleep (Armitage et al. 1994); this difference may be accounted for by the lower dose (20 mg) used in the study by Armitage et al. (1994).

The clinical significance of the eye movements in patients treated with fluoxetine is unclear, and unfortunately, too few patients on different doses were assessed to draw reliable conclusions about dose effect. In addition, the severity of depression pre- and posttreatment with fluoxetine at different doses was not measured for this group of depressed patients either. So, the extent to which eye movements are associated with antidepressant effect cannot be determined based on these data.

The presence of myoclonus in some of our patients treated with fluoxetine was expected, in that many antidepressants have been found to cause leg-jerking during sleep. However, the presence of clinically significant PLMD, defined in terms of specific periodicity and frequency criteria for the primary sleep disorder PLMD (Diagnostic Classification Steering Committee 1990) in a relatively large proportion $(4 \%$ ) of this sample of young depressed patients treated with fluoxetine was surprising, as PLMD is not frequently seen in young adults. It has been noted to occur in approximately $12 \%$ of those who complain of insomnia at a sleep disorders center (Coleman et al. 1982), a figure still lower than that observed in our group. Patients treated with fluoxetine in this study were not studied polysomnographically prior to treatment to rule out preexisting PLMD. Thus, one hypothesis considered was a possible relationship between affective disorder and PLMD, independent of medication. However, absence of evidence of significant PLMs in our group of depressed, unmedicated young adults suggests that this is not the case.

As noted, myoclonus has not been consistently defined in the literature. In several studies in which an association was made between antidepressants and myoclonus, PSG was used to define objectively the leg movements (Noble and Matthew 1969; Lippmann et al. 1977; Garvey and Tollefson 1987; Askenasy and Yahr 1988). Yet, periodicity and frequency criteria were not used or were very different from one another (Guilleminault et al. 1975; Ware 1983; Myers et al. 1986). Therefore, it is difficult to compare our findings with those of other studies that report antidepressant-related myoclonus. In addition to PLMD, we observed aperiodic myoclonic jerks in some of the fluoxetine patients. Although these movements were not included in our PLMD indices, they contributed to disruption of sleep in patients treated with fluoxetine. It is important to make distinctions between the various types of myoclonus that have been associated with antidepressant medication and PLMD, whose appearance exclusively during sleep and whose periodicity may constitute a unique phenomenon.

Several theories have been proposed to explain the mechanism of antidepressant-induced myoclonus, many of which implicate an excess of serotonin activity (Westheimer and Klawans 1974; Wong et al. 1974; Sheehan et al. 1980; Ware 1983). Fluoxetine is one of the first selective serotonin reuptake inhibitors described in the literature (Wong et al. 1974), and it is now widely used clinically. Our finding of clinically significant PLMD in a significant proportion of young adults treated with fluoxetine is consistent with hypotheses that implicate excessive serotonin as a neuropharmacological explanation for antidepressant-induced myoclonus.

In conclusion, the disruption of sleep continuity caused by frequent brief arousals during non-REM sleep and muscle tone increases, including greater likelihood of PLMD, may explain in part the frequent sleep complaints associated with fluoxetine. All these features may reflect an overall elevated level of arousal after treatment with fluoxetine. The significance of the increased frequency of eye movements is unclear, and the extent to which they may parallel treatment response should be explored. The serotonin selectivity of fluoxetine may be responsible for the increased eye activity, an "optical akathisia" that appears to occur during both relaxed wakefulness and sleep, as well as for increased overall muscle tone and PLMD. The mechanism that underlies these effects of fluoxetine and possibly other 
selective serotonergic reuptake inhibitor medications needs to be understood, especially in light of the growing clinical use of these drugs.

\section{ACKNOWLEDGMENTS}

The authors thank Daniel Buysse, M.D., for his advice on an earlier draft of this paper and James Hudson, M.D., for assistance in collecting control data. This work was supported in part by NIDA grant DA00115, NIMH grant MH 36224, and MH 49821.

\section{REFERENCES}

Armitage R, Trivedi M, Rush J, Pechacek P, Cole D, Dunnam D (1994): Effects of fluoxetine on EOG and EMG activity during sleep and depression. Sleep Res 23:61

Askenasy JM, Yahr MD (1988): Is monoamine oxidase inhibitor induced myoclonus serotonergically mediated? J Neural Transm 72:67-76

Atlas Task Force, Christian Guillemenault, Chairman (1992): EEG arousals: Scoring rules and examples. A preliminary report from the Sleep Disorders Atlas Task Force of the American Sleep Disorders Association. Sleep 15(2):174-184

Casas M, Garcia-Ribera C, Alyarez E, Udina C, MaQueralto J, MaGrau J (1987): Myoclonic movements as a sideeffect of treatment with therapeutic doses of clomipramine. Int Clin Psychopharmacol 2:333-336

Coleman RM (1982): Periodic movements in sleep (nocturnal myoclonus) and restless legs syndrome. In Guilleminault $C$ (ed), Sleeping and Waking Disorders: Indications and Techniques. Menlo Park, CA, Addison-Wesley, pp 265-295

Coleman RM, Roffwarg HP, Kennedy SV, et al. (1982): Sleepwake disorders based on a polysomnographic diagnosis-A national cooperative study. JAMA 247:997-1003

Cooper GL (1988): The safety of fluoxetine: An update. Br J Psychiatry 153(suppl. 3):77-86

Diagnostic Classification Steering Committee, Thorpy MJ, Chairman (1990): International Classification of Sleep Disorders: Diagnostic and Coding Manual. Rochester, MN, American Sleep Disorders Association

Dorsey CM, Cunningham SL, Lukas SE, Winkelman JW, Richardson GS, Jones K (1992a): Fluoxetine induced eve movements during non-REM sleep. Sleep Res 21:55

Dorsey CM, Cunningham SL, Richardson GS, Lukas SE, Winkelman JW (1992b): Increased risk of periodic leg movements during sleep with use of fluoxetine. Sleep Res 21:56

Evans DL, Davidson J, Raft D (1982): Early and late side effects of phenelzine. J Clin Psychopharmacol 2(3):208-210
Forstl H, Pohlmann-Eden B (1990): Amplitudes of somatosensory evoked potentials reflect cortical hyperexcitability in antidepressant-induced myoclonus. Neurology 40:924-926

Garvey MJ, Tollefson GD (1987): Occurrence of myoclonus in patients treated with cyclic antidepressants. Arch Gen Psychiatr 44:269-272

Guilleminault C, Raynal D, Weitzman ED, Dement WC (1975): Sleep-related periodic myoclonus in patients complaining of insomnia. Trans Am Neurol Assoc 100:19-22

Hamilton M (1969): A rating scale for depression. J Neurol Neurosurg Psychiatr 23:56-62

Keck PE, Hudson JI, Dorsey CM, Campbell PI (1991): Effect of fluoxetine on sleep [letter]. Biol Psychiatr 29:618-619

Lippmann S, Moskovitz R, O'Tuama L (1977): Tricyclicinduced myoclonus. Am J Psychiatr 134(1):90-91

Myers BA, Klerman GL, Hartmann E (1986): Nocturnal cataclysms with myoclonus: A new side effect of clomipramine. Am J Psychiatr 143(11):1490-1491

Noble J, Matthew H (1969): Acute poisoning by tricyclic antidepressants: Clinical features and management of 100 patients. Clin Toxicol 2:403-421

Rechtschaffen A, Kales AA (1968): A Manual of Standardized Terminology, Techniques, and Scoring for Sleep Stages of Human Subjects. Washington DC, Department of Health, Education, and Welfare

Schenck CS, Mahowald MW, Kim SW, O'Connor KA, Hurwitz TD (1992): Prominent eye movements during NREM sleep and REM sleep behavior disorder associated with fluoxetine treatment of depression and obsessive-compulsive disorder. Sleep 15:226-235

Sheehan DV, Claycomb JB, Kouretas N (1980): Monoamine oxidase inhibitors: Prescription and patient management. Int J Psychiatr 10(2):99-121

Spitzer RL, Williams JW, Gibbon M (1987): Structured Clinical Behavior for DSM-III-R. New York, Biometrics Research Division, New York State Psychiatric Institute

Stark P, Hardison CD (1985): A review of multicenter controlled studies of fluoxetine vs imipramine and placebo in outpatients with major depressive disorder. J Clin Psychiatr 46(3, Sect. 2):53-58

Ware JC (1983): Tricyclic antidepressants in the treatment of insomnia. J Clin Psychiatr 44(9, Sect 2):25-28

Wernicke JF (1985): The side-effect profile and safety of fluoxetine. J Clin Psychiatr 46(3, Sect. 2):59-67

Westheimer R, Klawans HL (1974): The role of serotonin in the pathophysiology of myoclonic seizures associated with acute imipramine toxicity. Neurology 24:1175-1177

Wong DT, Horng JS, Bymaster FP, Hauser KL, Molloy BB (1974): A selective inhibitor of serotonin uptake. Lilly 110140, 3-(p-trifluoromethylphenoxy)-N-methyl-3-phenylpropylamine. Life Sci 15:471-479 